\title{
MicroRNA-183-5p Promote Hepatocellular Carcinoma Proliferation Via Accelerating Pyroptosis By Targeting Fox01
}

Yu ling Chang

Gansu University of Chinese Medicine

Yaqian Niu

Gansu University of Chinese Medicine

Fang Liu

Gansu University of Chinese Medicine

Yan mei Song

Gansu university of Chinese Medicine

Huiyuan Chu

Gansu University of Chinese Medicine

Shisan Bao

The University of Sydney

Che Chen ( $\nabla$ chen72123@163.com )

Gansu University of Chinese Medicine https://orcid.org/0000-0003-3313-9554

\section{Research Article}

Keywords: Hepatocellular carcinoma, miR-183-5p, Pyroptosis, Fox01, NLRP3

Posted Date: September 20th, 2021

DOl: https://doi.org/10.21203/rs.3.rs-856242/v1

License: (9) (i) This work is licensed under a Creative Commons Attribution 4.0 International License.

Read Full License 


\section{Abstract}

Hepatocellular carcinoma (HCC) is the second leading cause of cancer worldwide, causing 700,000 deaths annually. Despite decades of extensive research of HCC, the prognosis of HCC remains unsatisfactory, mainly due to lack of reliable sensitive biomarkers. Therefore, it is fundamentally important to identify novel biomarkers for early diagnosis of HCC, as well as explore the underlying mechanisms of HCC progression. Pyroptosis, a highly inflammatory form of lytic programmed cell death, is attracting more attention in HCC. microRNAs are involved in the regulation of pyroptosis. miR-183-5p, an oncogene, is up-regulated in $\mathrm{HCC}$, but it is unclear the relationship between miR-183-5p and pyroptosis in HCC, which was investigated in vitro. Our data demonstrated that miR-183-5p promoted proliferation of HCC, accompanied with upregulating pyroptosis related molecules, as well as IL-1 $\beta /$ IL-18.

\section{Precis}

miR-183-5p promotes pyroptosis via targeting Fox01 in HCC HepG2 cells, consequently contributing to cell proliferation. Such finding may provide be a novel therapeutical specific target for treatment of HCC.

\section{Introduction}

Hepatocellular carcinoma (HCC), the main primary liver cancer, is health problem worldwide with unacceptable high mortality and poor prognosis [1]. There is great progression in treatment of HCC over recent years, the prognosis of HCC patients is still very poor, mainly due to these patients were at the advanced stage when diagnosed [2]. Furthermore, there are serious side effects but limited efficacy in many drugs for the treatment of HCC, and/or development of primary or secondary drug resistance $[3,4]$. Therefore, it is fundamentally important to identify novel biomarkers with great sensitive and specificity for early diagnosis of HCC. In addition, it should also explore the precise underlying mechanisms of HCC progression for development of novel therapeutic drugs.

Pyroptosis is a form of lytic programmed cell death initiated by inflammasomes, detecting cytosolic contamination or perturbation [5]. Pyroptosis drives activation of caspase-1 or caspase11/4/5, which cleave gasdermin $\mathrm{D}$, separating its $\mathrm{N}$-terminal pore-forming domain (PFD) from the $\mathrm{C}$-terminal repressor domain (RD), accompanied by the release of inflammatory factors, such as IL-1 $\beta$ and IL-18[6, 7]. More recently, pyroptosis has attracted attention for its role in anti-cancer treatments, particularly among these cancer patients developed apoptosis-related drug resistance[8, 9]. Therefore, exploring the inner link between pyroptosis and cancer is conducive to discovering new drugs for the treatment of cancer. It has been reported that pyroptosis is involved in development of HCC [10], perhaps involving microRNAs (miRNA),[11, 12].

miRNA, which is often combined with 3'untranslated region (3' UTR) of messenger RNA, is a type of endogenous non-coding single-stranded small RNA with a length of $19-25$ bases $[13,14]$. miRNA-183 family includes miR-183, miR-96 and miR-182 [15]. Abnormal expression of miR1835p is related to the 
pathogenesis of a number of malignancies [16-18]. miR-183 is significantly up-regulated in HCC via regulating transcription factors or the demethylation of the gene promoter region, compared with normal tissues ${ }^{[1,19]}$. Forkhead box protein 01 (Fox01) is a vital transcriptional factor involves oxidative stress via regulating anti-oxidases [20]. Fox01 has been identified as a co-target of miR-183-5p, miR-96-5p and miR-182-5p in cancers [20]. More recently it has been demonstrated that FoxO1 is involved in pyroptosis $[21,22]$.

Although microRNA involves in other diseases via regulating pyroptosis, the interaction between miR-183$5 p$ and pyroptosis in HCC is unclear. Here we investigated whether miR-183-5p regulates the development of HCC via pyroptosis.

\section{Materials And Methods}

\section{Cell Culture and source of miRNA}

Human HCC HepG2 cells used was purchased from the Cell Bank of the Chinese Academy of Sciences by Kilton Biotechnology Co., Ltd. (Shanghai, China). HepG2 cells were cultured in a humidified incubator with $37^{\circ} \mathrm{C}$ atmospheres supplemented with $5 \% \mathrm{CO}_{2}$, using Dulbecco's modified eagle's medium (DMEM) containing $10 \%$ fetal bovine serum (FBS) and 1\% penicillin and streptomycin. miR-183-5p mimics, mimics negative control (NC), miR-183-5p inhibitor, inhibitor NC were purchased from Jima Pharmaceutical Technology Co., Ltd. (Shanghai, China). The sequence was listed in Table I.

\section{Reagents and antibodies}

Lip-2000 were obtained from Jima Pharmaceutical Technology Co., Ltd. (Shanghai, China); ELISA reagent kit: Feiya Biological Technology Co., Ltd (Jiangsu, China); CCK-8 reagent kit: Yisheng Biotechnology Co., Ltd (Shanghai, China). Antibodies used for Western blot were as follows: Fox01, NLRP3, caspase-1, GSDMD, IL-1 $\beta$, IL-18 and GAPDH (GeneTex, USA; Servicebio, Wuhan, China). NLRP3, caspase-1, IL-1 $\beta$, IL18 and GAPDH were used in a dilution of 1:1,000. Fox01, GSDMD were used in a dilution of 1:500.

\section{Cell transfection}

HepG2 cells were plated in 6-well plate with $1 * 10^{6} / \mathrm{ml}$. miR-183-5p mimics, mimics NC or miR-183-5p inhibitor, or inhibitor NC were transfected into cells, according to the manufacturer's protocol. Firstly, configuration solution A ( $25 \mu \mathrm{L}$ liposome lip-2000) and solution B ( $10 \mu \mathrm{L}$ miR-183-5p mimics, mimics NC or miR-183-5p inhibitor, inhibitor NC) were added in $100 \mu \mathrm{L}$ serum-free basal medium, then were incubate at room temperature for $4 \mathrm{~h}$. The transfection medium was replaced with fresh DMEM medium containing $10 \%$ fetal bovine serum for further culture.

\section{Cell counting kit 8 (CCK-8)}

CCK-8 assay was performed according to the manufacturer's instructions. Briefly, HepG2 cells were plated in a 96-well plate with $1 * 10^{4} / \mathrm{mL}$, miR-183-5p mimics, mimics NC or miR-183-5p inhibitor, or inhibitor NC 
were transfected into cells, then $10 \mu \mathrm{L}$ of CCK-8 solution was added to each well of a 96-well plate and incubated at $37^{\circ} \mathrm{C}$ for $4 \mathrm{~h}$. The optical density was measured at an absorption wavelength of $450 \mathrm{~nm}$. Results were normalized to control levels.

\section{IL-1 $\beta$, IL-18 (ELISA)}

Culture medium was collected according to manufacturer's protocols, IL-1 $\beta$ or IL-18 in the supernatant was measured, using commercial ELSIA Assay Kit. In brief, IL-1 $\beta$, IL-18 substrate mix and assay buffer were added into cell culture supernatants in a 48 well plate. After the incubation at $37^{\circ} \mathrm{C}$ for $15 \mathrm{~min}$ without light, the absorbance was recorded at $450 \mathrm{~nm}$ on microplate reader.

\section{Quantitative real-time polymerase chain reaction (qRT-PCR)}

Total RNA was extracted from HCC cells, using TrizoL reagent according to the manufacturer's instruction. A total of $2 \mu \mathrm{g}$ of RNA was used for cDNA synthesis with a Reverse Transcription Kit in $20 \mu \mathrm{L}$ reaction volume. Equal amounts of cDNA were subjected to PCR with the SYBR Green PCR Master Mix Kit. The condition including an initial denaturation at $94^{\circ} \mathrm{C}$ for $5 \mathrm{~min}$, followed by 40 cycles of $94^{\circ} \mathrm{C}$ for 30 $\mathrm{s}, 60^{\circ} \mathrm{C}$ for $30 \mathrm{~s}, 72^{\circ} \mathrm{C}$ for $30 \mathrm{~s}$, and a terminal extension at $72^{\circ} \mathrm{C}$ for 5 min. Each sample was examined in triplicate and $\beta$-actin was used as the internal control. GraphPad Prism v8 was used to create the scatter plots. All primers were obtained from TaKaRa. The primers we used for qRT-PCR is listed in Table II.

\section{Western blot analysis}

Equal amount of proteins were extracted from cells and separated by sodium dodecyl sulphatepolyacrylamide gel electrophoresis (SDS-PAGE) according to the manufacturer's instruction, and then transferred to a polyvinylidene difluoride membrane. After blocking the membrane with $5 \%$ non-fat milk, membranes were incubated overnight with the specific primary antibodies at $4^{\circ} \mathrm{C}$, and then incubated for $1 \mathrm{~h}$ with the corresponding secondary antibody. The band intensities were analyzed and protein expression was quantified using Alpha EaseFC.

\section{Statistical analysis}

Statistically significant differences were assessed by Student's t-test or one-way analysis of variance tests followed by Bonferroni's method or Fisher's Least Significant Difference for multiple comparisons when necessary. The data were expressed as the mean \pm SD. Coefficients of correlation $(r)$ were determined by the Pearson correlation method. The criterion for statistical significance was set at $P<$ 0.05 .

\section{Results}

miR-183-5p promotes HCC cell proliferation 
The role of miR-183-5p in the proliferation of HCC was verified in HepG2 cells transfected with miR-183$5 p$ mimics, mimics NC, miR-183-5p inhibitor, or inhibitor NC, respectively. The expression of miR-183-5p was more than 10-fold higher in miR-183-5p mimics treated HepG2 cells, compared with mimics NC. In addition, the expression of miR-183-5p was suppressed by $90 \%$ in the HepG2 cells treated with miR-183$5 p$ inhibitor (Fig. 1A).

Using CCK kit, the proliferation rate of miR-183-5p mimics group was markedly higher than mimics NC group, following $24 \mathrm{~h}, 48 \mathrm{~h}$ or $72 \mathrm{~h}$ transfection; and miR-183-5p inhibitor group was visibly lower than inhibitor NC group. the results suggested that miR-183-5p can promote HCC proliferation (Fig. 1B).

\section{miR-183-5p promotes Fox01 expression.}

Bioinformatics demonstrated that MiR-183-5p targets Fox01. Thus qRT-PCR and Western Blot were used to determine the expression of FoxO1 after these different transfections for $24 \mathrm{~h}$ of HepG2 cells, as stated above (Fig. 2). The expression of Fox01 in miR-183-5p mimics group was significantly higher than that in mimics NC group; Compared with inhibitor NC, the expression of Fox01 in the miR-183-5p inhibitor group was significantly lower.

\section{miR-183-5p promote hepatocellular carcinoma pyroptosis}

It was investigated that the interaction between miR-183-5p and pyroptosis in HCC, qRT-PCR and Western blotting were used to determine the expression of pyroptosis related moleculars, NLRP3, caspase-1, GSDMD, IL-1 $\beta$ and IL-18 following the different transfections for $24 \mathrm{~h}$. Our results demonstrated that the expression of pyroptosis related mRNA NLRP3, caspase-1, GSDMD, IL-1 $\beta$ and IL-18 of miR-183-5p mimics group are significantly increased compared with the mimics NC group (Fig. 3). Conversely, compared with the inhibitor NC group, the expression of pyroptosis related mRNA of miR-183-5p inhibitor group are evidently reduced. Western blotting also confirmed similar changes at protein level.

Using ELISA, IL-1 $\beta$ and IL-18 were clearly higher in the supernatants from miR-183-5p mimics group, compared with mimics NC group (Fig. 4). IL-1 $\beta$ and IL-18 release from the miR-183-5p inhibitor group was significantly reduced, compared with inhibitor NC.

\section{Discussion}

In the current study, we have demonstrated that miRNA-183 contributes to the pyroptosis of HCC in vitro at mRNA and protein level, consequently contributing to cell proliferation.

A class of small non-coding RNAs with the ability to negatively regulate gene expression, called miRNAs, have been found to have a great effect on the progression of tumors [23, 24]. miRNAs involve in cancer cell differentiation and homeostasis, playing an indispensable role in many pathophysiological processes, including HCC [22]. It has been demonstrated that miR-183-5p, as an oncogene, is up-regulated in $\operatorname{HCC}[25,26]$. In our current study, it was explored whether miR-183-5p target Fox01 based on bioinformatic prediction. [12, 20,27]. Our study confirmed this prediciton, showing a linkage beteen miR- 
183-5p and FoxO1 HCC cells in vitro, suggesting that miR-183-5p promotes FoxO1 expression in HCC HepG2.

Studies have demonstrated that miRNAs are involved in the regulation of cell pyroptosis [28-31]. The importance of our finding is that pyroptosis is being focused in anti-cancer treatment, because emerging evidence suggests that cancer can evade apoptosis-induced death via resisting apoptotic signals [32, 33]. Therefore, it is critically important to develop new anti-cancer drugs based on non-apoptotic cell death. Increasing evidences have demonstrated that pyroptosis plays a dual role during tumor progression, including antitumor and tumor-promoting [34]. In addition, pyroptosis eliminates damaged cells, causing an inflammatory response to defend against intracellular infection [35]. On the other hand, the inflammatory micro-environment activated during the process of pyroptosis is auspicious to the occurrence and progress of cancers, which may stimulate autocrine or paracrine processes that favor carcinogenic inflammation, metastasis, angiogenesis and tumor growth [36, 37]. In the present research, we find that miR-183-5p can promote the expression of pyroptosis related molecular NLRP3, caspase-1, GSDMD, IL-1 $\beta$ and IL-18, and the release of inflammatory factor IL-1 $\beta$ and IL-18 in HCC HepG2 cells. Therefore, miR-183-5p can promote pyroptosis by targeting Fox01.

Cell proliferation is an important feature of hepatocellular carcinoma, which is regulated by a variety of signaling pathways. A better understanding of its mechanism will help improve cancer treatment [38, 39]. We demonstrated that miR-183-5p promoted the proliferation of HCC HepG2 cells, as well as pyroptosisrelated genes. This is in contrast with the finding by Fan et al. [40] showing that luteoloside (flavonoid), inhibits the proliferation, invasion and metastasis of HCC cells through inhibition of NLRP3 inflammasome mediated pyroptosis. Wan et al. [41] suggested miR-223 inhibited HCC cell proliferation by directly targeting NLRP3. Our explanation is that there are differential role of different miRNAs to malignant cells. However, there are opposite consequences about the effect of pyroptosis on the development of HCC. Zhang et al. revealed that AIF could suppress cell proliferation and metastasis by inducing pyroptosis-mediated activation of the NLRP3 inflammasome [42]. Berberine, a natural isoquinoline alkaloid, is reported to activate caspase-1 and has strong inhibitory effects on proliferation, and migration of HCCs in vitro experimental studies [43]. The tumor microenvironment is extremely intricate. The environment factors and gene signals experienced by the tumor microenvironment are very different, therefore it is not surprising that the response to the carcinogenic signal in these changing environments is also different. The molecular mechanism of the potential effect of pyroptosis on the proliferation of HCC needs further research in the future.

In conclusion, miR-183-5p is conducive to promoting pyroptosis by targeting FoxO1 in HCC HepG2 cells, thereby contributing to cell proliferation. This work provides a new idea for clinical treatment of hepatocellular carcinoma, establishing a certain of foundation for molecular targeted therapy. However, the present study has some limitations. Only one cell line, HepG2, was used in the experiments. Considering the different biological characteristics of HCC cell lines, different cell lines are needed to fully reveal the link between the miR-183-5p and pyroptosis. What's more, consider conducting in vivo experiments for further research to verify the in vitro results of this work. 


\section{Abbreviations}

HCC, hepatocellular carcinoma; NLRP3, nod-like receptor protein 3; PFD, pore-forming domain; RD, Repressor domain; IL, interleukin; Fox01, Forkhead box protein 01; TXNIP, thioredoxin interacting protein; ROS, reactive oxygen species; GSDMD, Gasdermin D.

\section{Declarations}

\section{Funding}

This research was supported by Lanzhou Cheng guan District Science and Technology Plan Project (Grant No. 2020JSCX0084) and Gansu Province Higher Education Research Project (Grant No. 2020-0405-JCC-1698).

\section{Availability of data and materials}

Not applicable.

\section{Acknowledgements}

The authors thank members of the clinical laboratory diagnostics teams in Gansu University of Chinese Medicine who gave their work in this study. This research was supported by Lanzhou Cheng guan District Science and Technology Plan Project(Grant No. 2020JSCX0084) and Gansu Province Higher Education Research Project (Grant No. 2020-0405-JCC-1698).

\section{Ethics approval and consent to participate}

Not applicable.

\section{Patient consent for publication}

Not applicable

\section{Authors' contribution}

This work was conceived and designed by Yuling Chang, Che Chen and Shisan Bao. The experiments were performed by Yuling Chang, Yaqian Niu, Fang Liu, Yanmei Song, and Huiyuan Chu. The manuscript was prepared by Yuling Chang and Yaqian Niu. Che Chen and Shisan Bao revised the manuscript; and all authors approved the final version of the manuscript.

\section{Conflicts of interest}

The authors declare no conflict of interest.

\section{References}


1. Liang Z, Gao Y, Shi W, Zhai D, Li S, Jing L, Guo H, Liu T, Wang Y, Du Z: Expression and significance of microRNA-183 in hepatocellular carcinoma. The Scientific World Journal (2013) 381874, 2013

2. Faivre S, Rimassa L, Finn RS (2020) Molecular therapies for HCC: Looking outside the box. J Hepatol 72:342-352

3. Hage C, Hoves S, Strauss L, Bissinger S, Prinz Y, Poschinger T, Kiessling F, Ries CH (2019) Sorafenib Induces Pyroptosis in Macrophages and Triggers Natural Killer Cell-Mediated Cytotoxicity Against Hepatocellular Carcinoma. Hepatology 4:1280-1297

4. Rebouissou S, Nault JC (2020) Advances in molecular classification and precision oncology in hepatocellular carcinoma. J Hepatol 2:215-229

5. Fang Y, Tian S, Pan Y, Li W, Wang Q, Tang Y, Yu T, Wu X, Shi Y, Ma P, Shu Y (2020) Pyroptosis: A new frontier in cancer. Biomed Pharmacother 121:109595

6. Kim DH, Kim SM, Lee B, Lee EK, Chung KW, Moon KM, An HJ, Kim KM, Yu BP, Chung HY (2017) Effect of betaine on hepatic insulin resistance through FOXO1-induced NLRP3 inflammasome. The Journal of Nutritional Biochemistry 45:104-114

7. Xu B, Jiang M, Chu Y, Wang W, Chen D, Li X (2018) Gasdermin D plays a key role as a pyrptosis executor of non-alcoholic steatohepatitis in humans and mice. J Hepatol 68:773-782

8. Ruan J, Wang S, Wang J (2020) Mechanism and regulation of pyroptosis-mediated in cancer cell death. Chemico Biological Interactions Journal 323:109052

9. Wang J, Li X, Liu Y, Peng C, Zhu H, Tu G, Yu X, Li Z (2020) CirchIPK3 Promotes Pyroptosis in Acinar Cells Through Regulation of the miR-193a-5p/GSDMD Axis. Frontiers in Medicine 7:88

10. Chu Q, Jiang Y, Zhang W, Xu C, Du W, Tuguzbaeva G, Qin Y, Li A, Zhang L, Sun G et al (2016) Pyroptosis is involved in the pathogenesis of human hepatocellular. Oncotarget 7:84658-84665

11. Li P, Zhong X, Li J, Liu H, Ma X, He R (2018) MicroRNA-30c-5p inhibits NLRP3 inflammasomemediated endothelial cell pyroptosis through $\mathrm{FOXO3}$ down-regulation in atherosclerosis. Biochem Biophys Res 4:2833-2840

12. Zeng R, Luo DX, Li HP, Zhang QS, Lei SS, Chen JH (2019) MicroRNA-135b alleviates MPP (+)mediated Parkinson's disease in in vitro model through suppressing Fox01-induced NLRP3 inflammasome and pyroptosis. Journal of Clinical Neuroscience 65:125-133

13. Murakami Y, Yasuda T, Saigo K, Urashima T, Toyoda H, Okanoue T, Shimotohno K (2006) Comprehensive analysis of microRNA expression patterns in hepatocellular carcinoma and nontumorous tissues. Oncogene 17:2537-2545

14. Wang X, Gong Y, Deng T, Zhang L, Liao X, Han C, Yang C, Huang J (2019) Diagnostic and prognostic significance of mRNA expressions of apolipoprotein $A$ and $C$ family genes in hepatitis $B$ virus-related hepatocellular carcinoma. J Cell Biochem 10:18246-18265

15. Bian W, Zhang H, Tang M, Zhang S, Wang L, Liu L, Wang W (2018) Potential Role of microRNA-183 as a Tumor Suppressor in Hepatocellular Carcinoma. cellular physiology biochemistry 5:2065-2072 
16. Miao F, Zhu J, Chen Y, Tang N, Wang X, Li X (2016) MicroRNA-183-5p promotes the proliferation, invasion and metastasis of human pancreatic adenocarcinoma cells. Oncology Letters 1:134-140

17. Zheng S, Zhong YF, Tan DM, Xu Y, Wang D (2019) miR-183-5p enhances the radioresistance of colorectal cancer by directly targeting ATG5. J Biosci 4:92

18. Li H, Pan X, Quan J, Li Z, Zhao L, Guan X, Xu J, Xu W, Lai Y (2019) Upregulation of miR-183-5p predicts worse survival in patients with renal cell cancer after surgery. Cancer Biomarkers 2:153-158

19. Anwar SL, Krech T, Hasemeier B, Schipper E, Schweitzer N, Vogel A, Kreipe H, Reena B (2017) hsa-mir183 is frequently methylated and related to poor survival in human hepatocellular carcinoma. World Journal of Gastroenterol 9:1568-1575

20. Liu Z, Du J, Ren J, Zhang Z, Guo X, Yan Y, Jia X, Gu N (2018) miR-183-96-182 clusters alleviated oxLDLinduced vascular endothelial cell apoptosis in vitro induced vascular endothelial cell apoptosis in vitro by targeting FOXO1. RSC Advances 8:135031-133504

21. Heo MJ, Kim TH, You JS, Blaya D, Sancho BP, Kim SG (2019) Alcohol dysregulates miR-148a in hepatocytes through Fox01, facilitating pyroptosis via TXNIP overexpression. Gut 4:708-720

22. Liu Y, Lou G, Li A, Zhang T, Qi J, Ye D, Zheng M, Chen Z (2018) AMSC-derived exosomes alleviate lipopolysaccharide/d-galactosamine-induced acute liver failure by miR-17-mediated reduction of TXNIP/NLRP3 inflammasome activation in macrophages. EBioMedicine 36:140-150

23. Bracken CP, Scott HS, Goodall GJ (2016) A network-biology perspective of microRNA function and dysfunction in cancer. Nat Rev Genet 12:719-732

24. Lin S, Gregory RI (2015) MicroRNA biogenesis pathways in cancer. Nat Rev Cancer 6:321-333

25. Szabo G, Bala S (2013) MicroRNAs in liver disease. Nature reviews 9:542-552

26. Wang XJ, Zhang DL, Fu C, Wei BZ, Li GJ (2016) MiR-183 modulates multi-drug resistance in hepatocellular cancer (HCC) cells via miR-183-IDH2/SOCS6-HIF-1 a feedback loop. European Review for Medical Pharmacological 10:2020-2027

27. Zhang L, Quan H, Wang S, Li X, Che X (2015) MiR-183 promotes growth of non-small cell lung cancer cells through FoxO1 inhibition. Tumour Biol 10:8121-8126

28. Gu C, Draga D, Zhou C, Su T, Zou C, Gu Q, Lahm T, Zheng Z, Qiu Q (2019) miR-590-3p Inhibits Pyroptosis in Diabetic Retinopathy by Targeting NLRP1 and Inactivating the NOX4 Signaling Pathway. Investigative Opthalmology Visual Science 13:4215-4223

29. Xu Q, Xing H, Wu J, Chen W, Zhang N: miRNA-141 Induced Pyroptosis in Intervertebral Disk Degeneration by Targeting ROS Generation and Activating TXNIP/NLRP3 Signaling in Nucleus Pulpous Cells. Frontiers in Cell and Developmental Biology 8, 2020

30. Tian BG, Hua Z, Wang ZJ, Li J (2020) Knockdown of microRNA-181a inhibits osteosarcoma cells growth and invasion through triggering NLRP3-dependent pyroptosis. European Review for Medical Pharmacological Sciences 24:7922

31. Jiang Z, Yao L, Ma H, Xu P, Li Z, Guo M, Chen J, Bao H, Qiao S (2017) miRNA-214 Inhibits Cellular Proliferation and Migration in Glioma Cells Targeting Caspase 1 Involved in Pyroptosis. Oncol Res 
6:1009-1019

32. Ashrafizadeh M, Zarrabi A, Hushmandi K, Hashemi F, Hashemi F, Samarghandian S, Najafih M (2020) MicroRNAs in cancer therapy: Their involvement in oxaliplatin sensitivity/resistance of cancer cells with a focus on colorectal cancer. Life Sci 256:117973

33. Yang JD, Heimbach JK (2020) New advances in the diagnosis and management of hepatocellular carcinoma. BMJ 371:m3544

34. Xia X, Wang X, Cheng Z, Qin W, Lei L, Jiang J, Hu (2019) The role of pyroptosis in cancer: pro-cancer or pro-“host”? Cell Death Dis 9:650

35. Kovacs SB, Miao EA (2017) Gasdermins: Effectors of Pyroptosis. Trends Cell Biol 9:673-684

36. Barber G, Anand A, Oficjalska K, Phelan JJ, Heeran AB, Flis E, Clarke NE (2020) Characterizing caspase-1 involvement during esophageal disease progression. Cancer Immunology Immunotherapy 69:2635-2649

37. Zitvogel L, Kepp O, Galluzzi L, Kroeme G (2012) Inflammasomes in carcinogenesis and anticancer immune responses. Nature immunology 13:343-352

38. Wang X, Lu J, Cao J, Ma B, Gao C, Qi F (2018) MicroRNA-18a promotes hepatocellular carcinoma proliferation, migration, and invasion by targeting Bcl2L10. Onco Targets Ther 11:7919-7934

39. Zhu J, Thompson CB (2019) Metabolic regulation of cell growth and proliferation. Nat Rev Mol Cell Biol 20:436-450

40. Sarkar D, Fan S, Wang Y, Lu J, Zheng Y, Wu D, Zhang Z, Cheng W (2014) Luteoloside Suppresses Proliferation and Metastasis of Hepatocellular Carcinoma Cells by Inhibition of NLRP3 Inflammasome. PLoS ONE 9:e89961

41. Wan L, Yuan X, Liu M, Xue B (2017) miRNA-223-3p regulates NLRP3 to promote apoptosis and inhibit proliferation of hep3B cells. Experimental Therapeutic Medicine 15:2429-2435

42. Zhang Y, Yang H, Sun M, He T, Liu Y, Yang X, Shi X, Liu X (2020) Alpinumisoflavone suppresses hepatocellular carcinoma cell growth and metastasis via NLRP3 inflammasome-mediated pyroptosis. Pharmacol Rep 72:1370-1382

43. Jin H, Jin X, Cao B, Wang W (2017) Berberine affects osteosarcoma via downregulating the caspase$1 / \mathrm{IL}-1 \beta$ signaling axis. Oncol Rep 37:729-736

\section{Tables}

Table l: miR-183-5p mimics, mimics NC, miR-183-5p inhibitor and inhibitor NC sequence. 


\begin{tabular}{|c|c|}
\hline miRNA & Sequence $(5 \rrbracket \rightarrow 3 \rrbracket)$ \\
\hline \multirow[t]{2}{*}{ miR-183-5p mimics } & sense: 5『UAUGGCACUGGUAGAAUUCACU3囚 \\
\hline & antisense: 5هUGAAUUCUACCAGUGCCAUAUU3』 \\
\hline \multirow[t]{2}{*}{ mimics NC } & sense: 5囚UUCUCCGAACGUGUCACGUTT3』 \\
\hline & antisense: ACGUGACACGUUCGGAGAATT3】 \\
\hline miR-183-5p inhibitor & 5囚AGUGAAUUCUACCAGUGCCAUA3\ \\
\hline inhibitor NC & 5『CAGUACUUUUGUGUAGUACAA3》 \\
\hline
\end{tabular}

Table II: miR-183-5p, U6, Fox01, NLRP3, caspase-1, GSDMD, IL-1 $\beta$ and IL-18 primer sequence.

\begin{tabular}{|c|c|}
\hline Gene & Primer sequence \\
\hline \multirow[t]{2}{*}{ miR-183-5p } & Forward冈GCGGCTATGGCACTGGTAGAA \\
\hline & Reverse®GTGCAGGGTCCGAGGTATTC \\
\hline \multirow[t]{2}{*}{ U6 } & Forward囚CTTAGTTGCGTTACACCCTTTCTTG \\
\hline & Reverse®CTGTCACCTTCACCGTTCCAGTTT \\
\hline \multirow[t]{2}{*}{ Fox01 } & Forward冈GATGGTCAAGAGCGTGCCCTA \\
\hline & Reverse $₫ T G G A T T G A G C A T C C A C C A A G A A$ \\
\hline \multirow[t]{2}{*}{ NLRP3 } & Forward囚GATCTTCGCTGCGATCAACA \\
\hline & Reverse『GGGATTCGAAACACGTGCATTA \\
\hline \multirow[t]{2}{*}{ caspase-1 } & Forward冈GCCTGTTCCTGTGATGTGGAG \\
\hline & Reverse TGCCCACAGACATTCATACAGTTTC \\
\hline \multirow[t]{2}{*}{ GSDMD } & Forward囚TGAATGTGTACTCGCTGAGTGTGG \\
\hline & Reverse冈CAGCTGCTGCAGGACTTTGTG \\
\hline \multirow[t]{2}{*}{ IL-1 $\beta$} & Forward冈CCAGGGACAGGATATGGAGCA \\
\hline & Reverse $\triangle T T C A A C A C G C A G G A C A G G T A C A G$ \\
\hline \multirow[t]{2}{*}{ IL-18 } & Forward囚CTGCCACCTGCTGCAGTCTA \\
\hline & Reverse TCTACTGGTTCAGCAGCCATCTTTA \\
\hline
\end{tabular}

Figures 
A

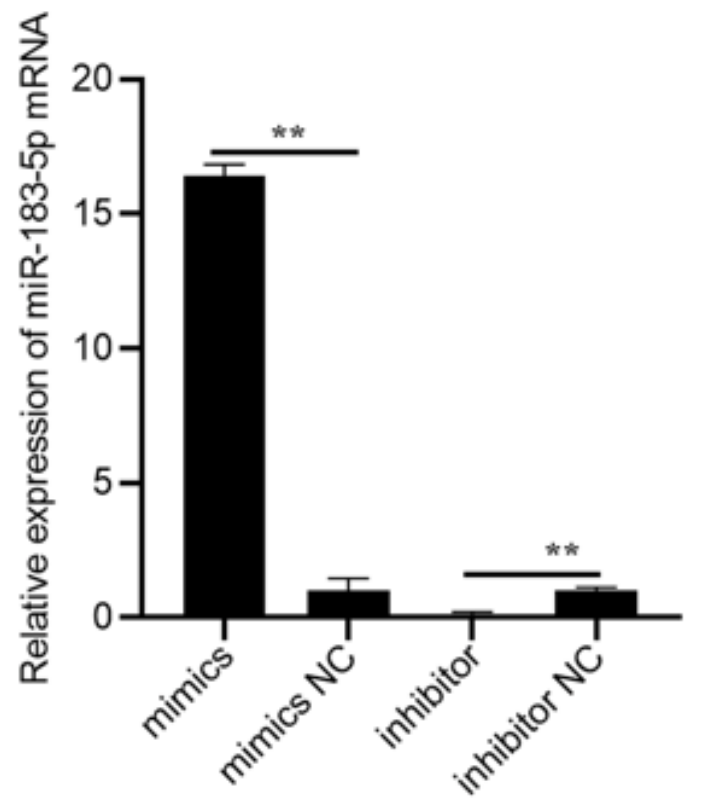

B $24 \mathrm{~h}$

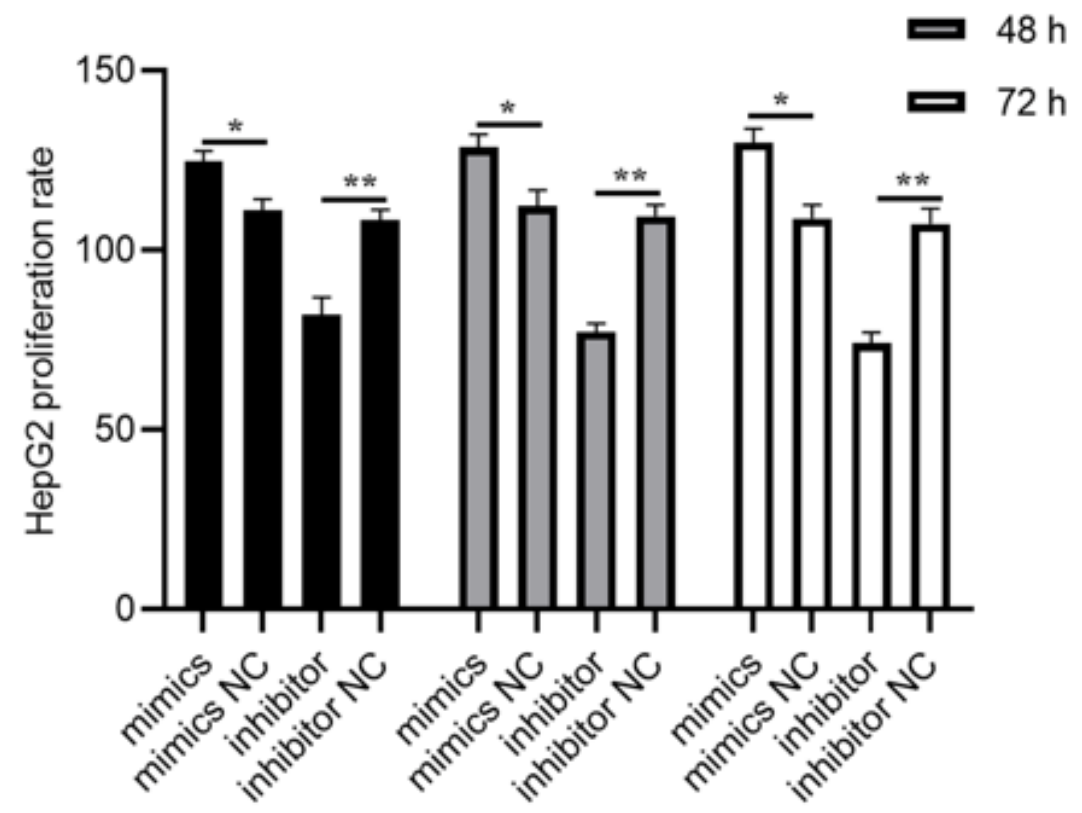

Figure 1

miR-183-5p promote HCC HepG2 cell proliferation. A. The relative expression of miR-183-5p in HepG2 cells after transfection, ${ }^{\star *} \mathrm{p}<0.01$. B. HCC proliferation rate after transfection $24,48,72 \mathrm{~h}$. At the same point, the miR-183-5p mimics compared with the mimics NC. The miR-183-5p inhibitor compared with the inhibitor $N C,{ }^{*} p<0.05,{ }^{* *} p<0.01$.
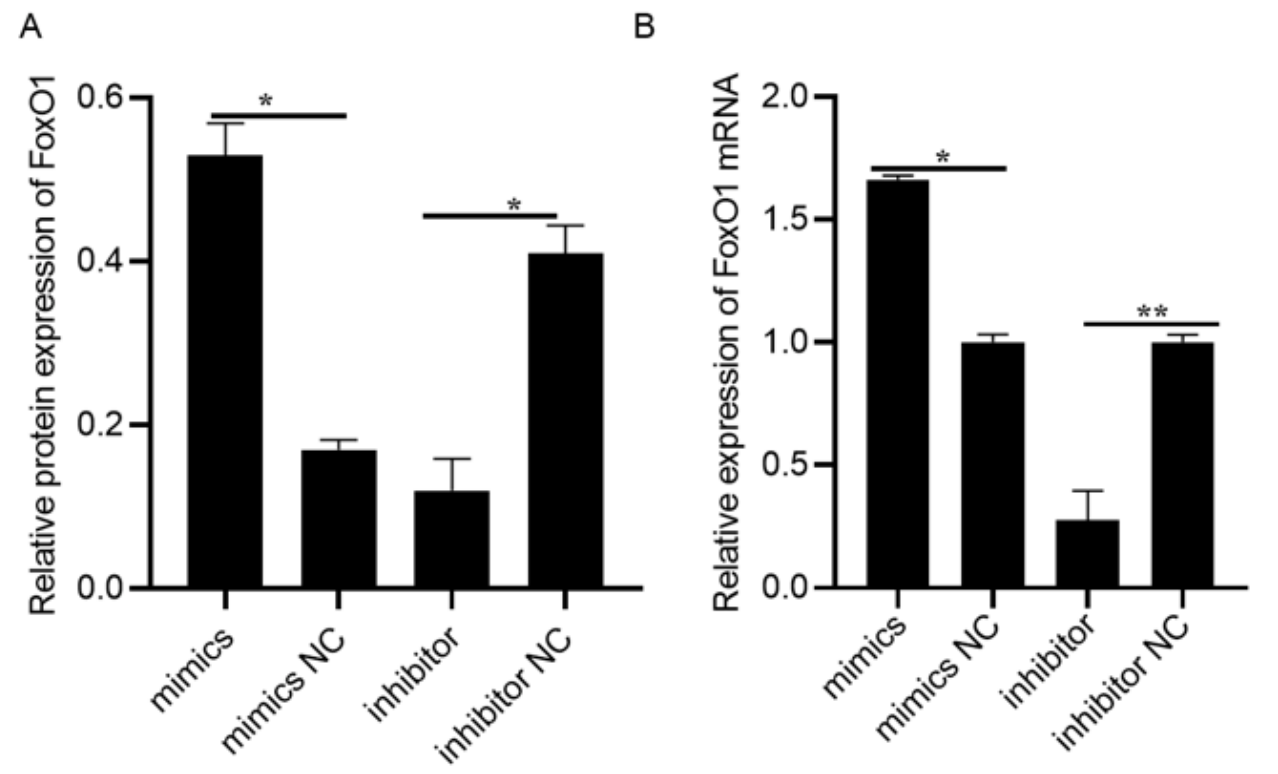

C

Figure 2

miR-183-5p promote Fox01 expression. A. The relative mRNA expression of Fox01 in HepG2 cells after transfection. B. The relative protein expression of Fox01 in HepG2 cells after transfection. C. 
Immunoblottings bands for Fox01 after transfection. ${ }^{*} \mathrm{p}<0.05,{ }^{*} \mathrm{p}<0.01$.

$\mathbf{A}$


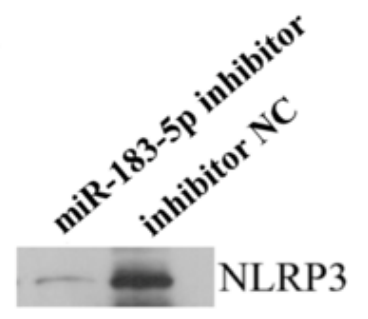

- caspase-1
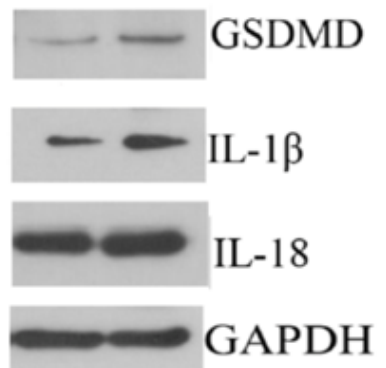

B



C

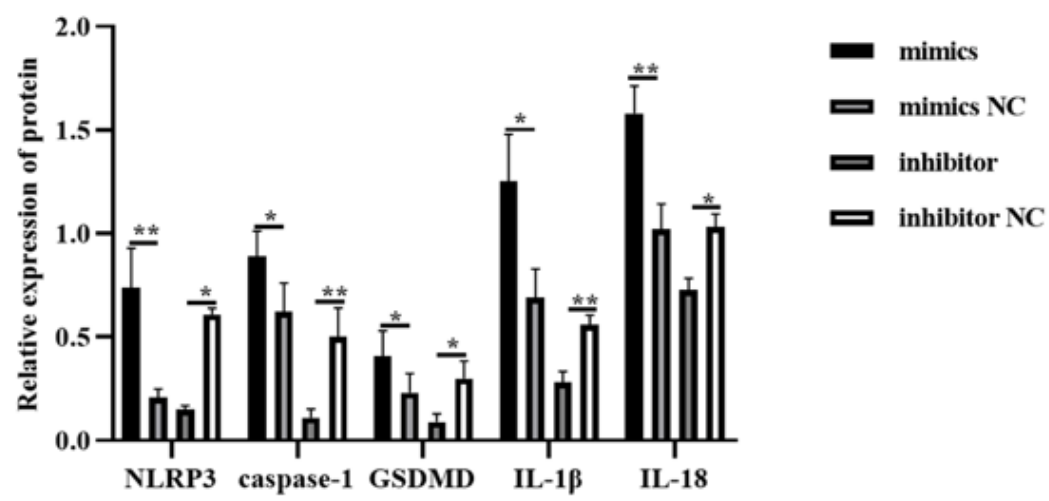

Figure 3

miR-183-5p promote the expression of pyroptosis related molecular. A. Immunoblottings bands for pyroptosis related protein after transfection. B. The relative mRNA expression of pyroptosis related gene in HepG2 cells after transfection. C. The relative protein expression of pyroptosis related gene in HepG2 cells after transfection. ${ }^{*} \mathrm{p}<0.05^{*},{ }^{*} \mathrm{p}<0.01$. 
A

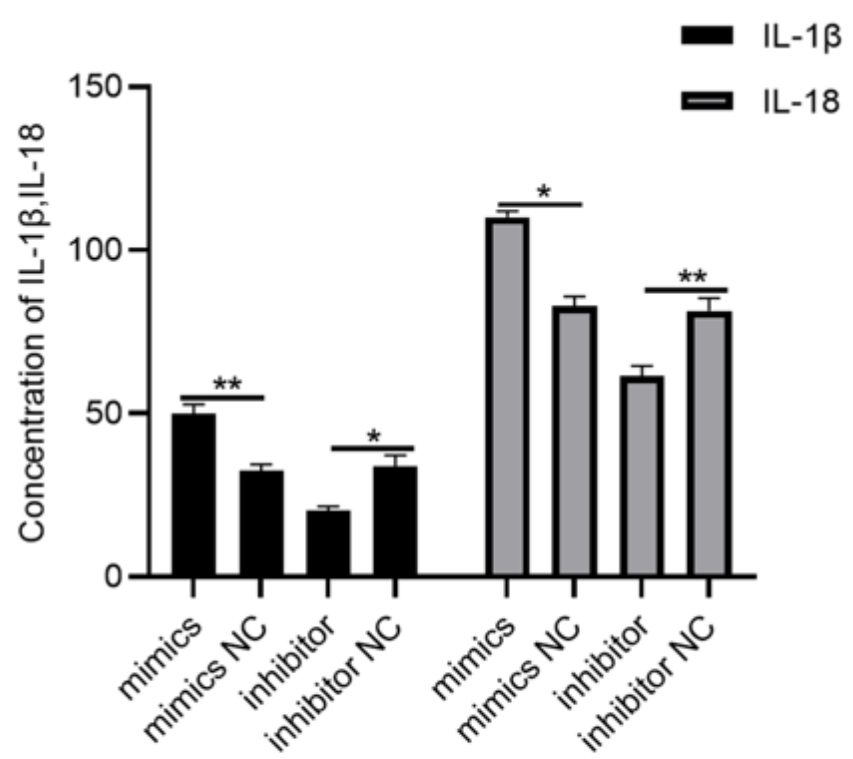

B

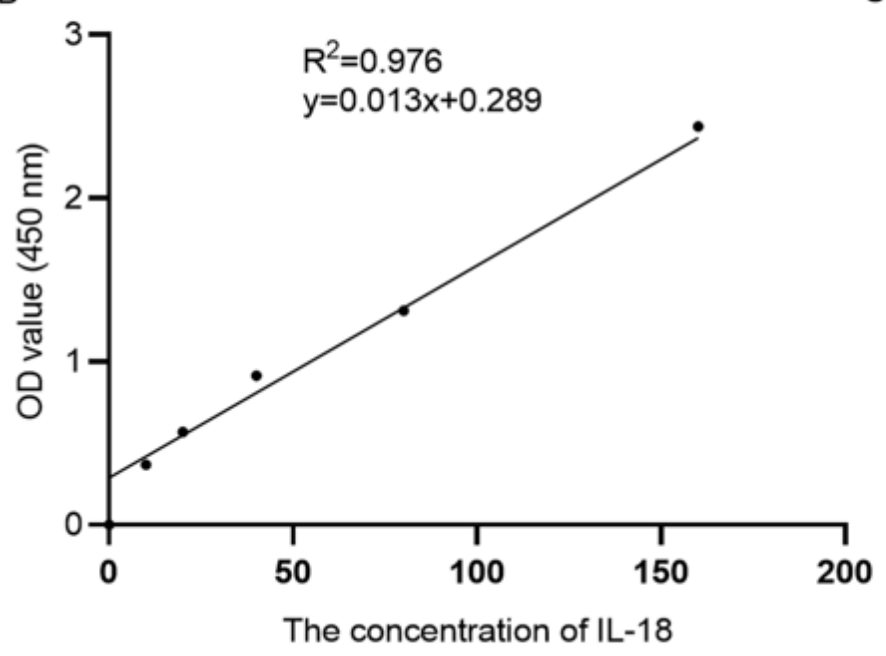

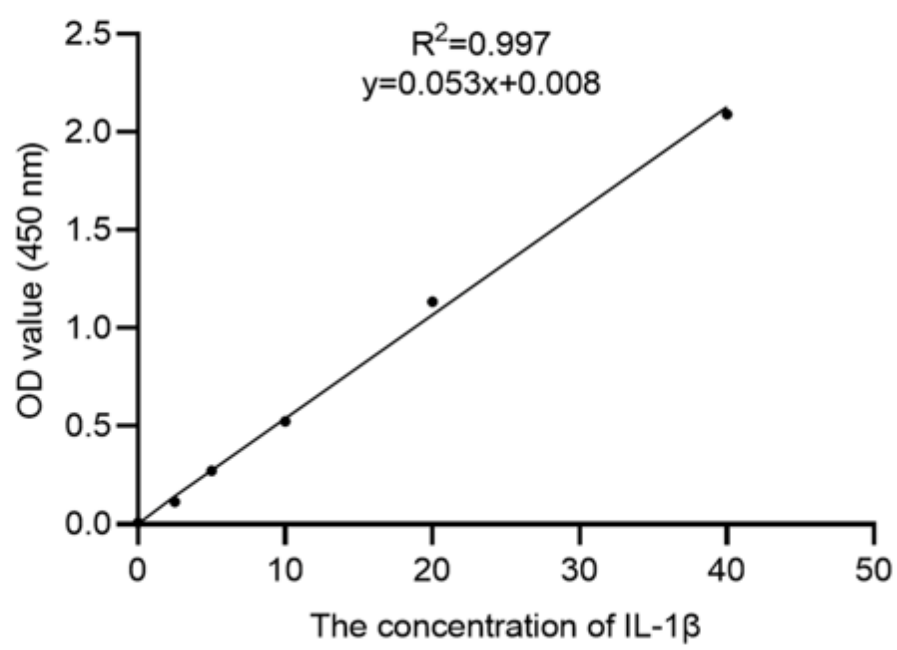

Figure 4

miR-183-5p promote the release of IL-1 $\beta$ and IL-18 in HepG2 cells after transfection. A. The release of IL$1 \beta$ and IL-18 in HepG2 cells after transfection. B. The standard curve line of IL-1 $\beta$. C. The standard curve line of IL-18. ${ }^{*} p<0.05,{ }^{* \star} p<0.01$. 